\title{
EFEITos do ACúmulo de DePósitos EM VÁlvUlas de AdMissão naS Emissões Legisladdas de POLUENTES
}

\author{
Rogério Nascimento de Carvalho ${ }^{1}$, Eurico da Silva Mello Neto ${ }^{1}$ \\ e Ricardo Almeida Barbosa de Sá ${ }^{1}$ \\ ${ }^{1}$ Petróleo Brasileiro S.A. \\ E-mails: rogerio.carvalho@ petrobras.com.br, e.mello@petrobras.com.br, \\ rsa@petrobras.com.br
}

\section{RESUMO}

Este artigo apresenta um estudo experimental para relacionar diferentes níveis de depósitos nas válvulas de admissão de um motor de combustão interna, com as emissões dos poluentes legislados no Brasil. Foram avaliados os níveis de 100 e $300 \mathrm{mg}$ de depósitos que correspondem respectivamente aos valores definidos pela Agência Nacional de Petróleo, Gás Natural e Biocombustíveis (ANP) para a aprovação das gasolinas aditivadas e para os depósitos a serem gerados por uma gasolina de referência. Também foi avaliado o nível de $500 \mathrm{mg}$ para a aplicação de técnicas estatísticas e de regressão linear.

Foi implantado um procedimento para a geração em banco de provas dos depósitos nas válvulas de admissão de um motor do modelo especificado pela norma NBR 16038 e para a medição em dinamômetro de chassi, das emissões de THC, $\mathrm{CO}$ e $\mathrm{NOx}$, em um veículo equipado com o mesmo modelo de motor, seguindo a norma NBR 6601.

Os resultados indicaram que não houve diferenças significativas para as emissões de THC e NOx, variando-se os depósitos nas válvulas nos níveis de 150 mg, $340 \mathrm{mg}$ e 519 mg. Com relação às emissões de CO, ao variar os depósitos entre 150 e 340 mg e entre 340 e 519 mg, também não foram encontradas diferenças estatisticamente significativas, porém a comparação entre os níveis de $150 \mathrm{mg}$ e 519 mg resultou em aumento da ordem de $31 \%$.

\section{INTRODUÇÃO}

Em 2013, a Agência Nacional de Petróleo, Gás Natural e Biocombustíveis (ANP) publicou a Resolução $\mathrm{n}^{\mathrm{o}} 40$ [1] na qual regula as especificações das gasolinas comerciais de uso automotivo, válidas a partir de $1^{\underline{0}}$ de janeiro de 2014 . As principais alterações definidas foram a redução do teor de enxofre de $800 \mathrm{ppm}$ para $50 \mathrm{ppm}$, os novos limites dos teores de hidrocarbonetos olefínicos e aromáticos, respectivamente em $25 \%$ e $35 \%$ em volume, e a alteração da temperatura de ponto final de ebulição de $220^{\circ} \mathrm{C}$ para $215^{\circ} \mathrm{C}$.

Nesta mesma resolução, foi estabelecido que toda gasolina $\mathrm{C}$ comercializada no território nacional a partir de $1^{\circ}$ de julho de 2015 , deverá conter aditivo detergente-dispersante, com o objetivo de controlar a formação de depósitos no motor. Atualmente está em discussão a postergação desta obrigatoriedade para 2017. Os critérios de aprovação das gasolinas 
aditivadas nesses requisitos foram estabelecidos pela Resolução ANP $\mathrm{n}^{-0} 1$ de 2014 [2] e apontam para a definição do limite máximo de $100 \mathrm{mg}$ de depósito médio nas válvulas de admissão.

O procedimento de medição dos depósitos em válvulas de admissão é regido pela norma NBR 16038 [3] e a efetividade dos aditivos deverá ser comprovada a partir de uma gasolina de referência. A especificação desta gasolina de referência, segundo a Resolução ANP $\mathrm{n}^{0} 1$ de 2014 [2] indica que ela deverá produzir um mínimo de $300 \mathrm{mg}$ de depósito médio nas válvulas de admissão.

Para melhor conhecer as potenciais vantagens da redução dos depósitos de motor, foi realizado este estudo para estabelecer a relação entre os níveis de $100 \mathrm{mg}$ e $300 \mathrm{mg}$ de depósitos médios nas válvulas de admissão e as respectivas emissões legisladas de gases poluentes.

Neste sentido, foi elaborado um procedimento de ensaios em motor e veículo capaz de formar, em banco de provas, os níveis de depósitos requeridos e sequencialmente medir os níveis de emissões de hidrocarbonetos (THC), monóxido de carbono (CO) e óxidos de nitrogênio (NOx), em dinamômetro de chassi, segundo a norma NBR 6601 [4].

\section{REVISÃO BIBLIOGRÁFICA}

A literatura especializada apresenta alguns estudos experimentais relacionando os efeitos dos depósitos em motor nas emissões de poluentes.

HOUSER e CROSBY [5] publicaram um artigo relacionando o impacto dos depósitos em válvulas de admissão nas emissões de THC, CO e NOx, a partir de medições realizadas em uma frota de táxis. Foram utilizados 20 veículos idênticos, para acúmulo de cerca de 80.000 $\mathrm{km}$, abastecidos com diferentes gasolinas com e sem aditivos.

Os níveis de depósitos foram estabelecidos seguindo somente os critérios de avaliação visual do Coordinating Research Council - CRC, recebendo notas entre 6 e 9 nas escalas de demérito, que definem o grau 10 para válvulas totalmente isentas de depósitos. Não foram feitas as pesagens das válvulas.

Os resultados deste estudo levaram a relações lineares de redução das emissões de forma proporcional a menores níveis de depósitos em válvulas de admissão (maiores valores para o CRC IVD Rating), como pode ser observado nas reproduções apresentadas nas figuras 1 a 3.

Nota-se que nos três casos (figuras 1 a 3), as inclinações das linhas de tendência (linhas contínuas) foram fortemente influenciadas pelos resultados correspondentes a níveis de depósitos nas válvulas de admissão menores que 7, que correspondem aos motores com maiores quantidades de depósitos formados. Entretanto, considerando-se somente os casos com níveis acima de 7 , as regressões lineares (linhas tracejadas) indicam que aparentemente não se pode supor variação das emissões, principalmente de $\mathrm{HC}$ e CO. 


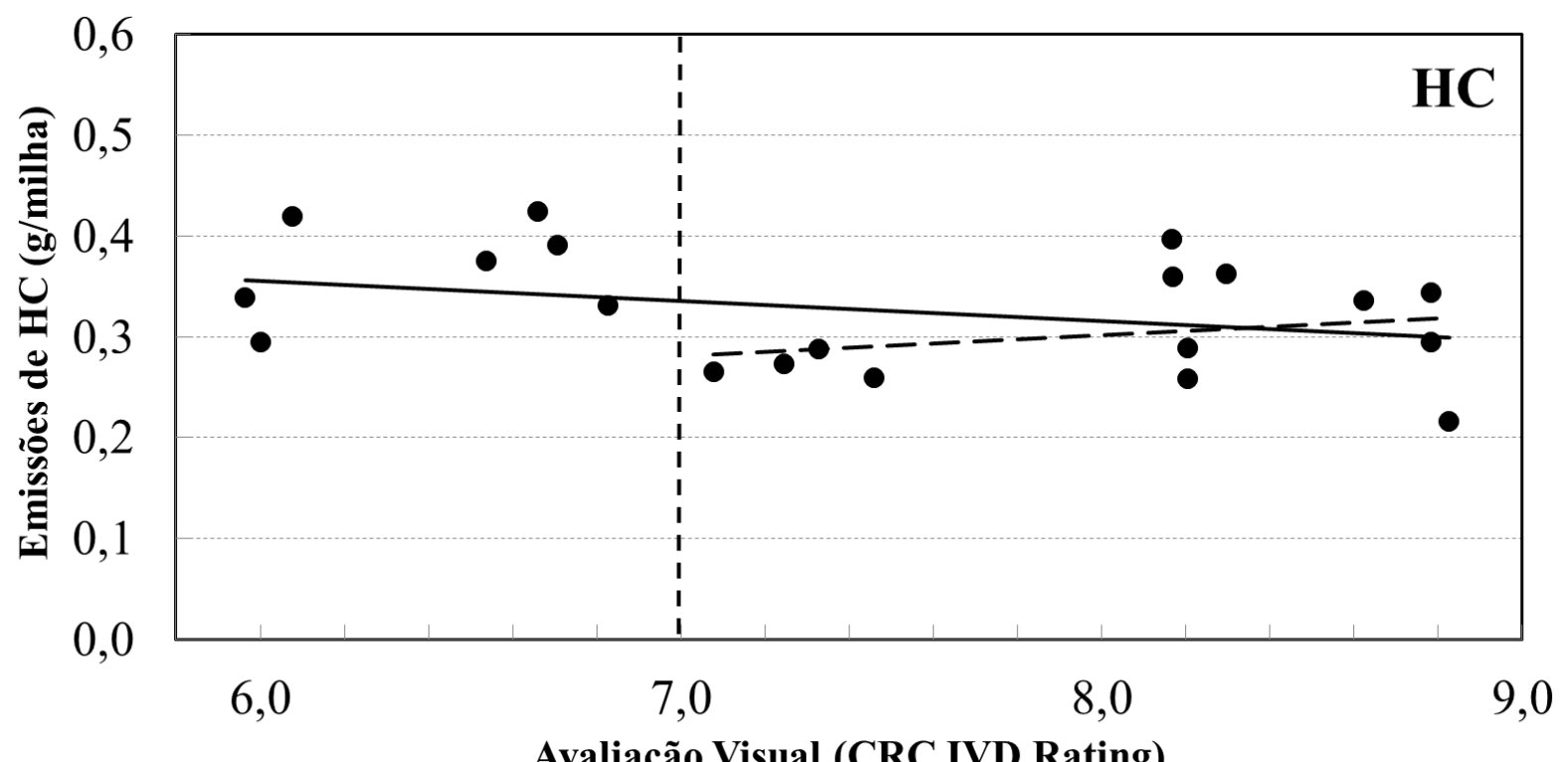

Figura 1. Relação das emissões de hidrocarbonetos (HC) com depósitos em válvulas de admissão (CRC IVD Rating) (adaptado de HOUSER \& CROSBY [5]).

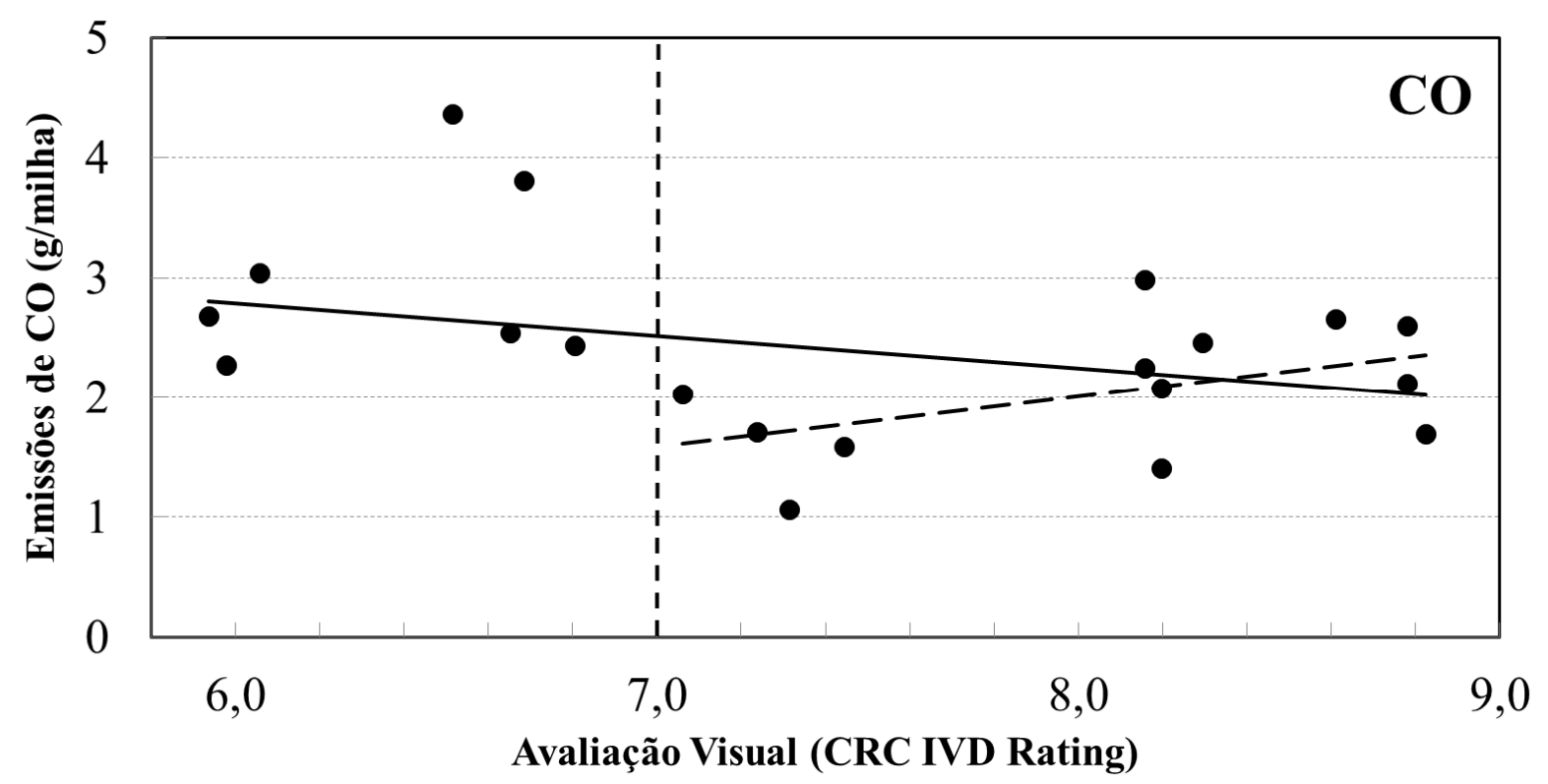

Figura 2. Relação das emissões de monóxido de carbono (CO) com depósitos em válvulas de admissão (CRC IVD Rating) (adaptado de HOUSER \& CROSBY [5]). 


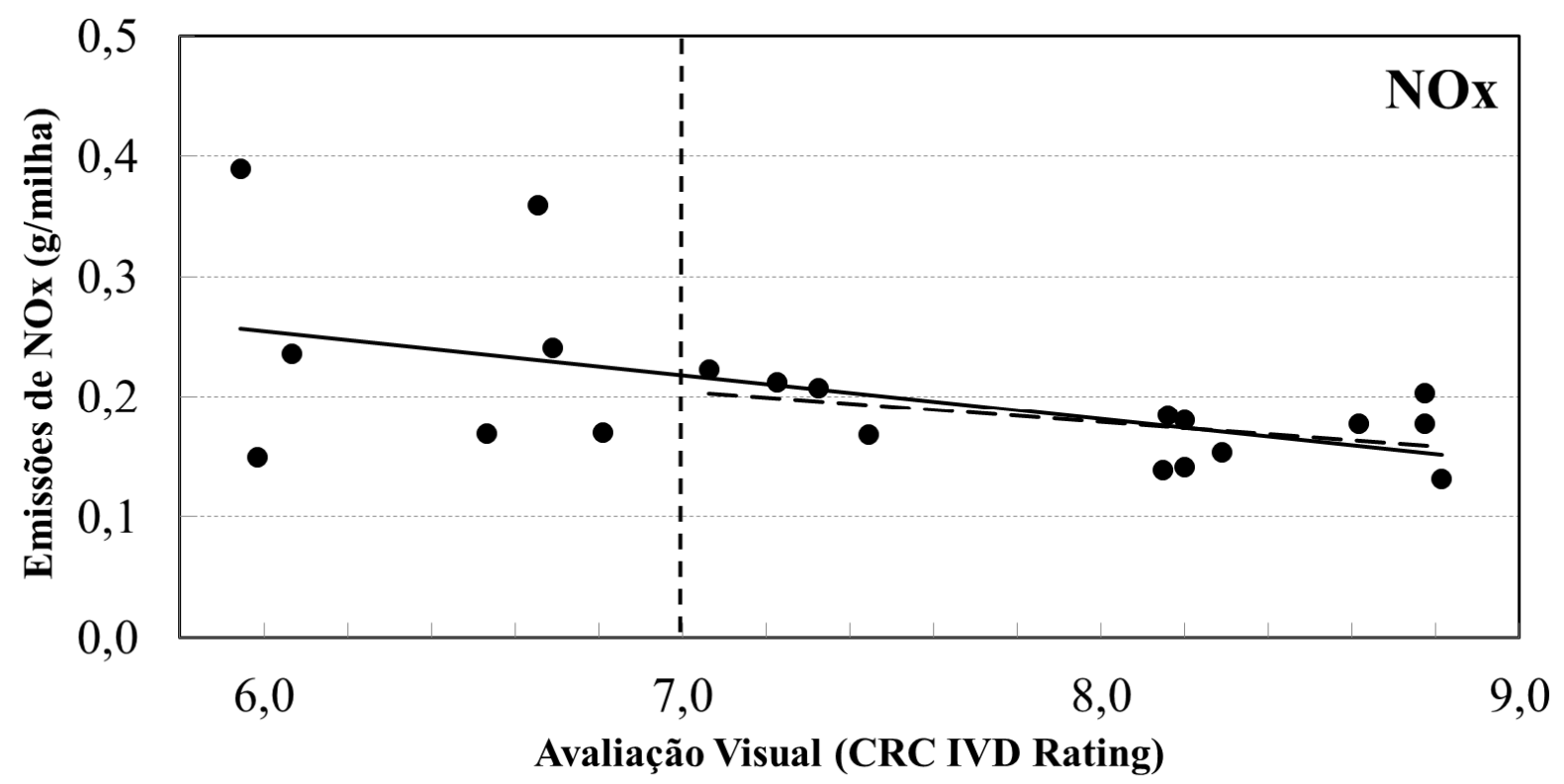

Figura 3. Relação das emissões de óxidos de nitrogênio (NOx) com depósitos em válvulas de admissão (CRC IVD Rating) (adaptado de HOUSER \& CROSBY [5]).

Para buscar relacionar os níveis de análises visuais segundo os padrões do CRC com as massas de depósitos nas válvulas de admissão, foi proposta uma correlação com base em cerca de 200 ensaios de diferentes combustíveis que compõem o banco de dados de depósitos em válvulas do Centro de Pesquisas e Desenvolvimento da Petrobras, como mostra a figura 4.

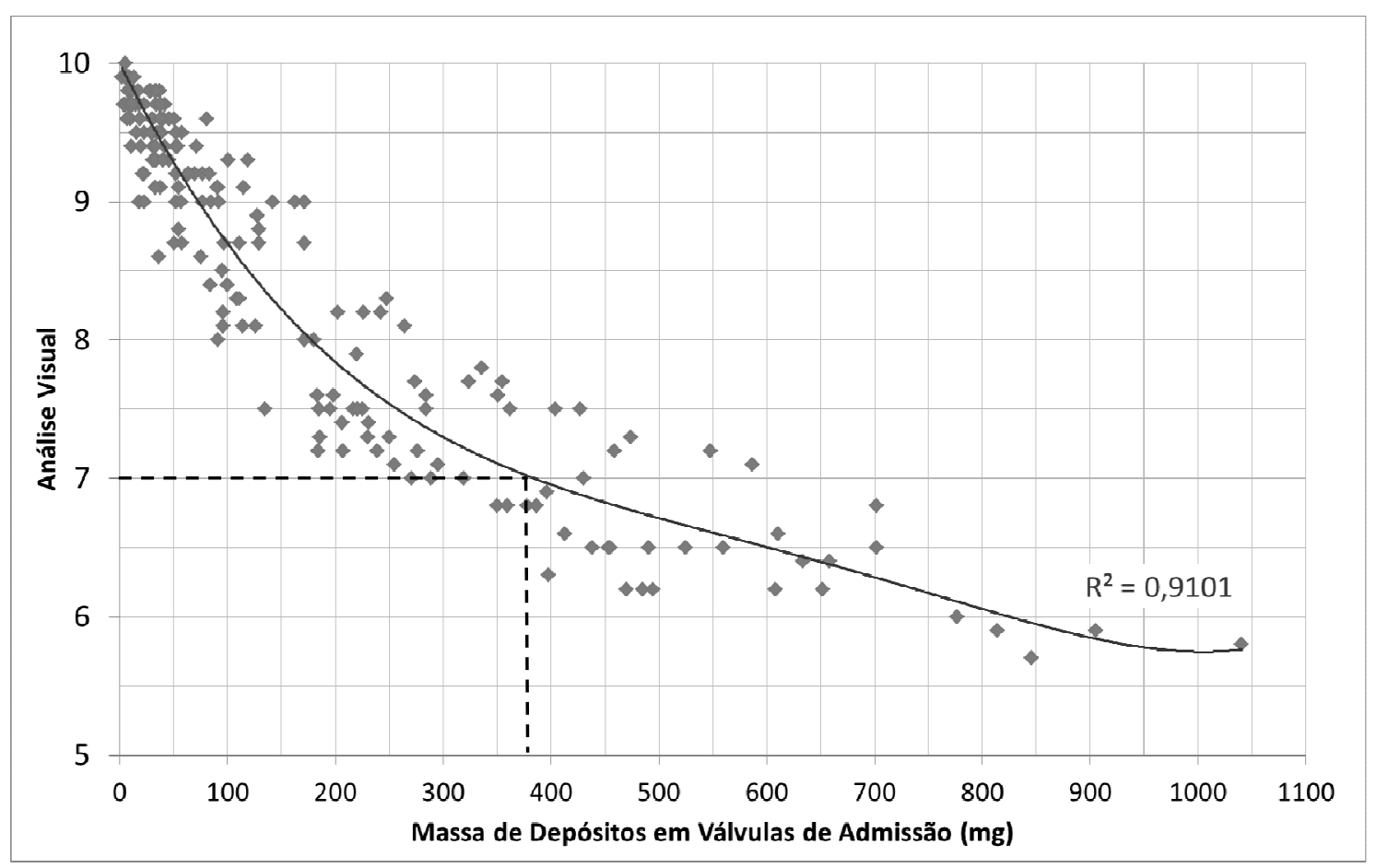

Figura 4. Relação entre análise visual e massa de depósitos em válvulas de admissão. 
Desta relação pode-se inferir que análises visuais com nota 7 de demérito correspondem a ensaios com cerca de $380 \mathrm{mg}$ de depósitos médios formados nas válvulas de admissão. Cabe salientar que a análise visual é subjetiva e realizada por comparação com padrões, servindo apenas como referência e informação adicional aos resultados dos ensaios.

Em outro estudo, ZAHALKA et al.[6] apresentam as relações entre os níveis de depósitos em válvulas de admissão e em câmaras de combustão com as emissões de HC, CO e NOx. Foram avaliados 20 veículos, que acumularam cerca de $40.000 \mathrm{~km}$ em rodagem de campo, com 6 diferentes tipos de gasolinas, incluindo combinações de gasolinas convencionais e reformuladas com 3 diferentes tipos de aditivos.

Nas figuras $5 \mathrm{a}$ e $5 \mathrm{~b}$, estão respectivamente reproduzidos os gráficos com os níveis de depósitos em válvulas de admissão e em câmaras de combustão, nos quais a sigla "RFG" representa a gasolina reformulada e a sigla "Conv", a convencional, sendo ainda as letras " $\mathrm{X}$ ", "Y", e "Z", os códigos correspondentes aos três diferentes aditivos utilizados.

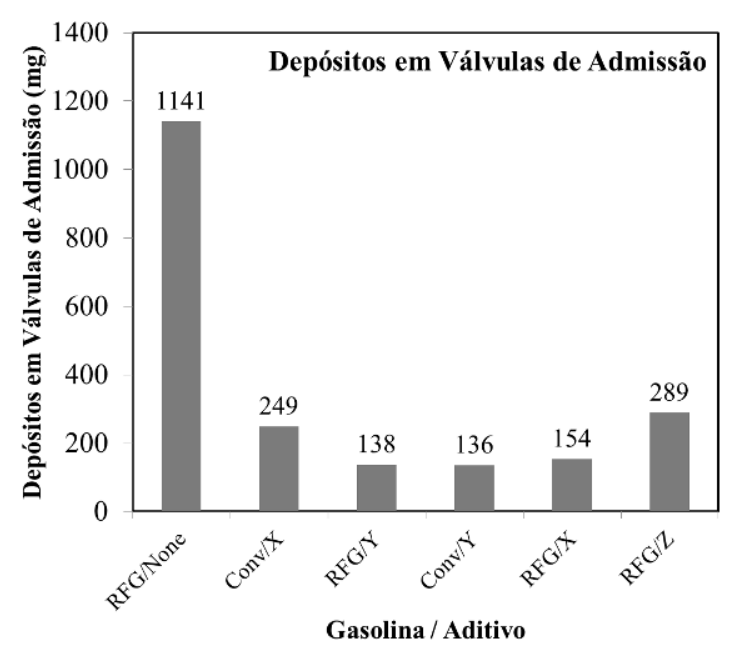

(a)

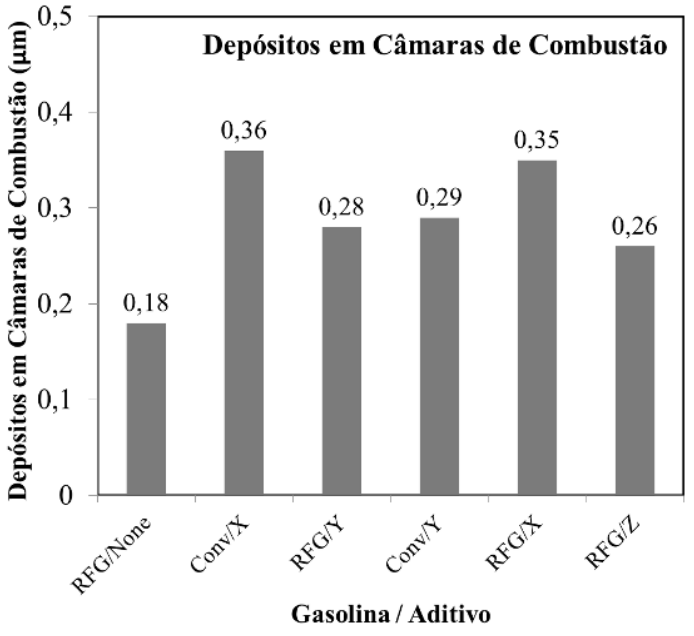

(b)

Figuras 5. (a) Depósitos médios nas válvulas de admissão e (b) depósitos médios nas câmaras de combustão com diferentes gasolinas e aditivos (adaptados de ZAHALKA et al.[6]).

Pode-se observar das figuras $5 \mathrm{a}$ e $5 \mathrm{~b}$ que a gasolina sem aditivo (RFG/None) promoveu um nível de depósitos em válvulas de admissão muito superior a todas as demais que continham aditivos. Em contrapartida, os depósitos em câmaras de combustão foram consistentemente maiores com as gasolinas aditivadas em relação à gasolina sem aditivos.

Nas figuras 6 a 8 estão reproduzidos os gráficos que relacionam respectivamente as emissões de HC, CO e NOx com as seis gasolinas ensaiadas. Nestes gráficos são apresentados os níveis de aumento das emissões medidas ao final do acúmulo de quilometragem em comparação ao início. 


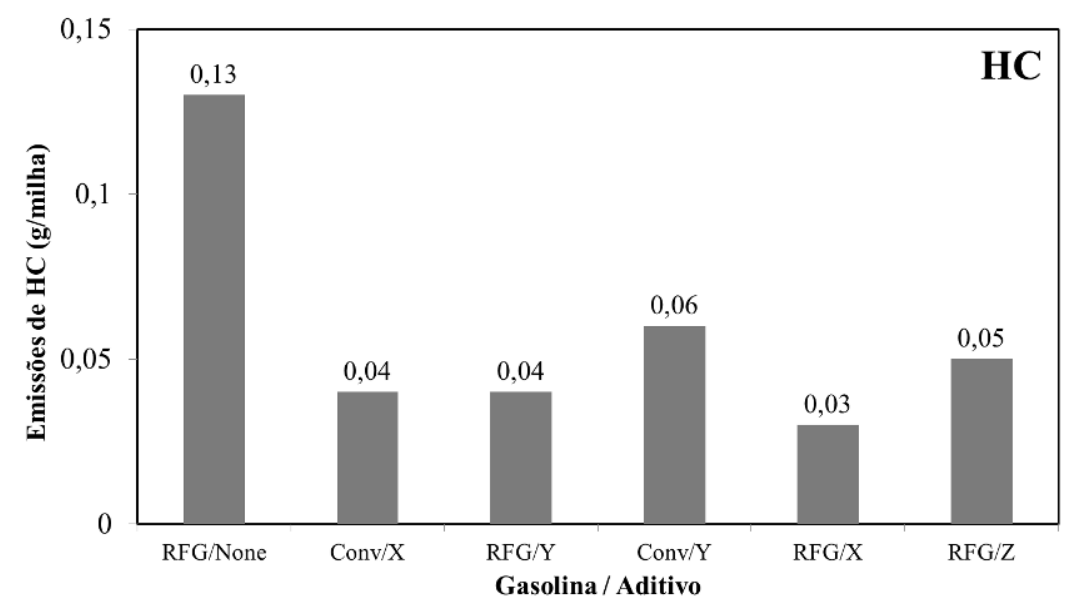

Figura 6. Aumento das emissões de HC após o acúmulo de 40.000 km. (adaptado de [6]).

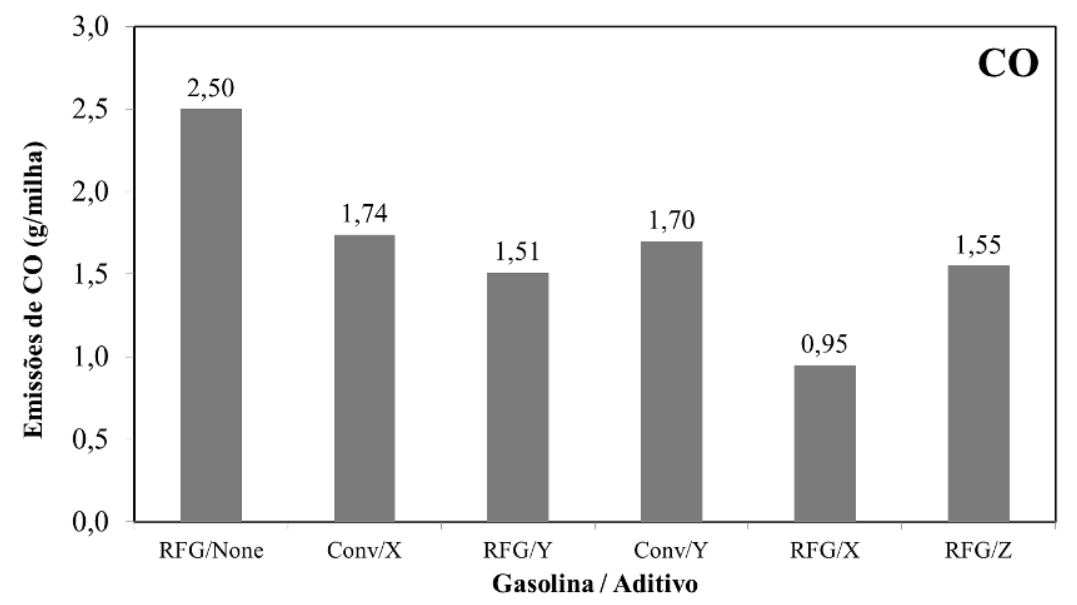

Figura 7. Aumento das emissões de CO após o acúmulo de 40.000 km. (adaptado de [6]).

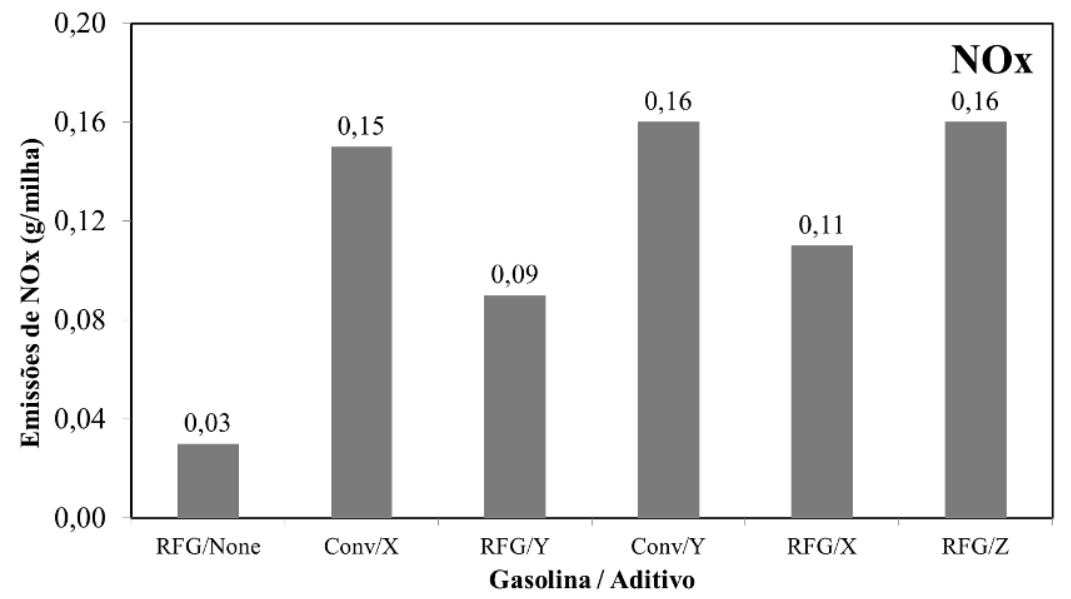

Figura 8. Aumento das emissões de NOx após o acúmulo de 40.000 km. (adaptado de [6]). 
Pode-se observar que o aumento das emissões de $\mathrm{HC}$ e $\mathrm{CO}$ ao final da rodagem foram inferiores nos veículos abastecidos com gasolinas aditivadas (depósitos em válvulas entre 136 mg e $289 \mathrm{mg}$ ) em comparação aos com gasolina não aditivada (1.141 mg). Em relação ao NOx, os autores atribuem o aumento das emissões aos correspondentes aumentos nos níveis dos depósitos em câmaras de combustão promovidos pelos aditivos utilizados, como se pode notar comparando-se as figuras 8 e $5 b$.

Alguns outros trabalhos relacionando as emissões de poluentes aos níveis de depósitos em motor foram encontrados na literatura [7-12] e, de forma geral, apresentam conclusões semelhantes. Estes trabalhos pontuam a tendência de aumento das emissões de $\mathrm{HC}$ e $\mathrm{CO}$ com maiores níveis de depósitos em válvulas de admissão, o aumento das emissões de NOx em função de maiores níveis de depósitos nas câmaras de combustão e mostram a eficácia dos aditivos na mitigação dos depósitos em válvulas, tendo porém o efeito colateral de aumentar os depósitos em câmaras de combustão.

\section{METODOLOGIA EXPERIMENTAL}

O objetivo deste estudo foi estabelecer uma relação experimental entre as emissões dos poluentes legislados no Brasil com os níveis de 100 mg e 300 mg de depósitos em válvulas de admissão.

Para tanto, foi estabelecido um protocolo de ensaios composto por procedimentos de acúmulo dos depósitos em banco de provas de motor e posteriormente testes de emissões de poluentes em dinamômetro de chassi. Adicionalmente aos níveis de $100 \mathrm{mg}$ e $300 \mathrm{mg}$ foi também avaliado o motor com $500 \mathrm{mg}$ de depósitos nas válvulas de admissão, buscando obter correlações com pelo menos três pontos de observação.

A etapa para acúmulo dos depósitos em válvulas de admissão foi realizada seguindo o procedimento estabelecido pela norma NBR 16038. Porém a parada dos ensaios não foi realizada nas 100 horas definidas pela norma, mas a partir de avaliações visuais das válvulas através de um boroscópio, equipamento dotado de sonda com câmera que possibilita a observação no interior do motor sem precisar desmontá-lo.

Os ensaios de emissões em dinamômetro de chassi foram realizados seguindo a norma NBR 6601 e foram medidas as emissões de hidrocarbonetos totais (THC), monóxido de carbono (CO) e óxidos de nitrogênio (NOx).

Foram utilizados o motor Fiat Tetrafuel que é definido como padrão pela norma NBR 16038 e um veículo Fiat Siena Tetrafuel, equipado com o mesmo modelo de motor. Em função de atualmente só haver procedimento disponível para este modelo de motor, não foram realizadas medições em outros veículos e motores.

As figuras 9 e 10 mostram, respectivamente, o motor instalado no banco de provas e o veículo de testes no dinamômetro de chassi. 


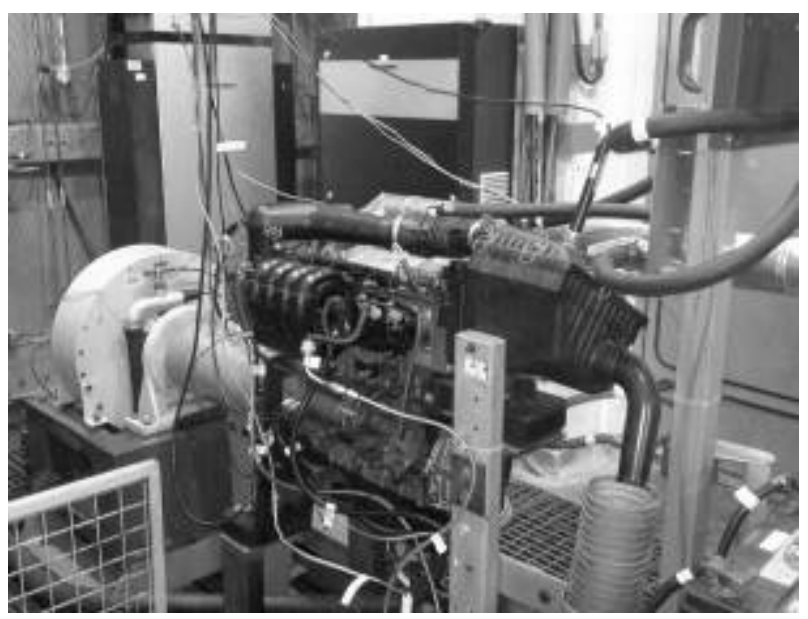

Figura 9. Motor Fiat Tetrafuel em banco de provas para acúmulo de depósitos em válvulas.

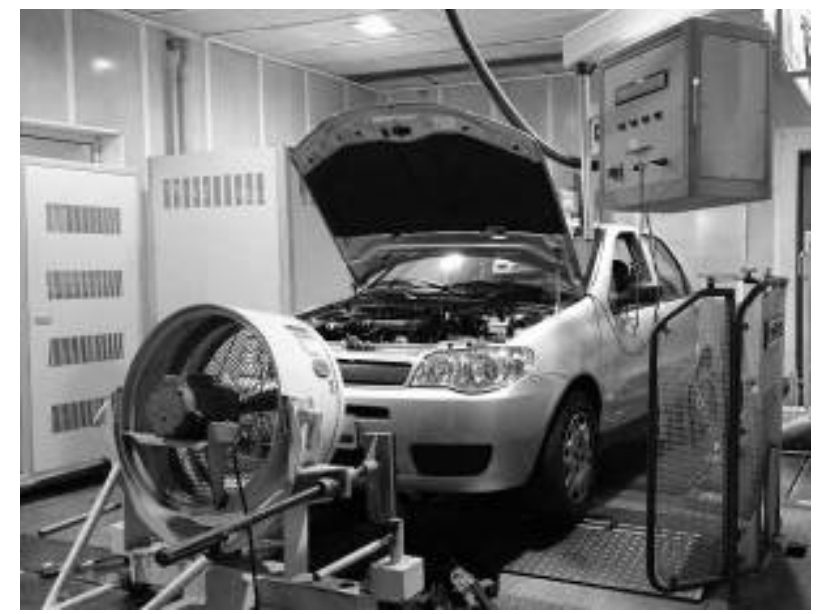

Figura 10. Veículo Fiat Siena Tetrafuel em dinamômetro de chassi para ensaios de emissões.

Desta forma, o seguinte procedimento de testes foi executado:

1. Desmontagem do cabeçote do motor do veículo de teste;

2. $\quad$ Limpeza das válvulas de admissão;

3. Montagem do cabeçote no motor do banco de provas para acúmulo de depósitos no nível de $100 \mathrm{mg}$, com parada determinada por avaliação visual através de boroscópio;

4. Desmontagem do cabeçote do motor do banco de provas e montagem no motor do veículo;

5. Execução dos ensaios de emissões em dinamômetro de chassi com $100 \mathrm{mg}$;

6. Desmontagem do cabeçote do motor do veículo;

7. Pesagem e avaliação das válvulas de admissão;

8. Montagem das válvulas de admissão no cabeçote, sem remoção dos depósitos;

9. Montagem do cabeçote no motor do banco de provas para acúmulo de depósitos no nível de $300 \mathrm{mg}$, com parada determinada por avaliação visual através de boroscópio; 
10. Desmontagem do cabeçote do motor do banco de provas e montagem no motor do veículo;

11. Execução dos ensaios de emissões em dinamômetro de chassi com 300 mg;

12. Desmontagem do cabeçote do motor do veículo;

13. Pesagem e avaliação das válvulas de admissão;

14. Montagem das válvulas de admissão no cabeçote, sem remoção dos depósitos;

15. Montagem do cabeçote no motor do banco de provas para acúmulo de depósitos no nível de $500 \mathrm{mg}$, com parada determinada por avaliação visual através de boroscópio;

16. Desmontagem do cabeçote do motor do banco de provas e montagem no motor do veículo;

17. Execução dos ensaios de emissões em dinamômetro de chassi com 500 mg;

18. Desmontagem do cabeçote do motor do veículo;

19. Pesagem e avaliação das válvulas de admissão.

Nos ensaios de acúmulo de depósitos foi utilizada uma gasolina C comercial contendo $25 \%$ em volume de etanol anidro (EAR) e os ensaios de emissões foram realizados com uma gasolina $\mathrm{C}$, com $22 \%$ de EAR, atendendo às especificações de gasolina padrão para ensaios de emissões.

Este procedimento permitiu avaliar o impacto nas emissões de níveis diferentes de depósitos nas válvulas de admissão. Entretanto, não foi possível relacionar os depósitos nas câmaras de combustão, uma vez que a parte inferior do motor do veículo, incluindo cilindros e pistões não foi submetida ao procedimento de acúmulo de depósitos.

Os ensaios de emissões de poluentes foram realizados em repetições até que pelo menos cinco resultados válidos de THC, $\mathrm{CO}$ e NOx fossem obtidos, atendendo ao critério de intervalo de confiança de 95\%, no qual os limites mínimo e máximo de controle são definidos como sendo distantes das médias das distribuições normais pela distância de 1,96 desvios-padrão.

A comparação entre as médias das emissões de cada poluente obtidas para os níveis de 100 mg e $300 \mathrm{mg}$ de depósitos em válvulas de admissão foram realizadas a partir do teste $t$ de Student, com nível de confiança de $95 \%$ (valor- $p \geq 0,05$ ).

Para a verificação de tendências de variação das emissões em função dos depósitos em válvulas, foram acrescentados os resultados das medições com $500 \mathrm{mg}$ e realizada a Análise de Variâncias (ANOVA). Quando a ANOVA indicou influência dos níveis de depósitos nas emissões a um nível de confiança de 95\%, foi gerada a linha de tendência a partir do ajuste por regressão linear.

\section{RESULTADOS}

Como mencionado, se objetivou realizar os ensaios de emissões com o acúmulo de depósitos médios nas válvulas de admissão nos níveis de $100 \mathrm{mg}, 300 \mathrm{mg}$ e $500 \mathrm{mg}$. Uma vez que o critério de parada do acúmulo de depósitos foi dado por observação através de boroscópio, não seria possível se obter os valores nominais exatos. Ainda assim, as pesagens das válvulas realizadas ao final dos ensaios de emissões de cada etapa, indicaram os valores médios 
respectivos de $150 \mathrm{mg}, 340 \mathrm{mg}$ e $519 \mathrm{mg}$, sendo considerados aceitos e representativos de cada um dos níveis estabelecidos.

A Tabela I apresenta os valores dos pesos de cada válvula e os respectivos pesos médios de cada ensaio.

Tabela I. Resultados dos ensaios para acúmulo dos depósitos nas válvulas de admissão.

\begin{tabular}{|c|c|c|c|c|c|}
\hline \multirow{2}{*}{ Teste } & \multicolumn{5}{|c|}{ Massa dos Depósitos nas Válvulas de Admissão (mg) } \\
\cline { 2 - 6 } & válvula 1 & válvula 2 & válvula 3 & válvula 4 & média \\
\hline $\mathbf{1 0 0}$ & 139,1 & 215,9 & 89,1 & 157,7 & 150 \\
\hline $\mathbf{3 0 0}$ & 357,1 & 330,4 & 310,9 & 360,3 & 340 \\
\hline $\mathbf{5 0 0}$ & 597,2 & 422,6 & 528,9 & 527,5 & 519 \\
\hline
\end{tabular}

As figuras 11 e 12 apresentam os registros fotográficos das válvulas após os acúmulos de 340 $\mathrm{mg}$ e $519 \mathrm{mg}$.
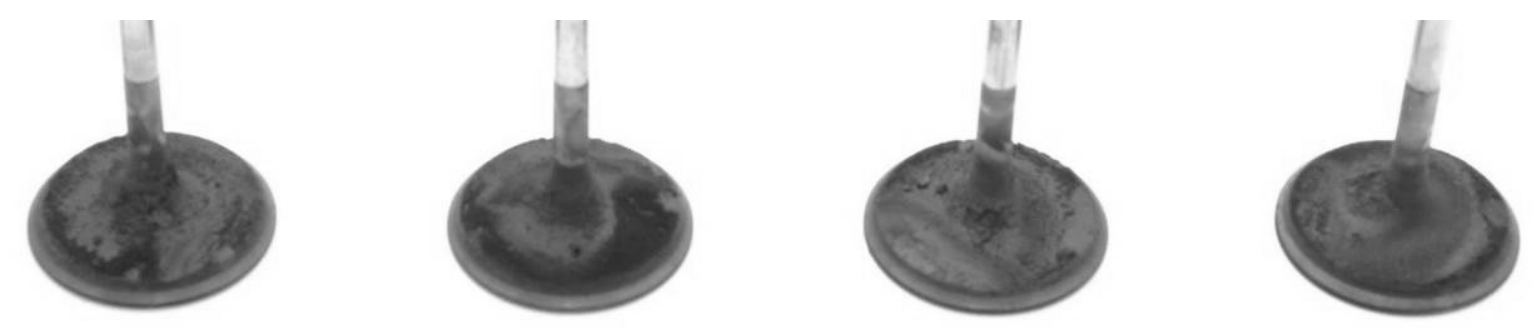

Figura 11. Registro fotográfico das válvulas de admissão após acúmulo de 340 mg.
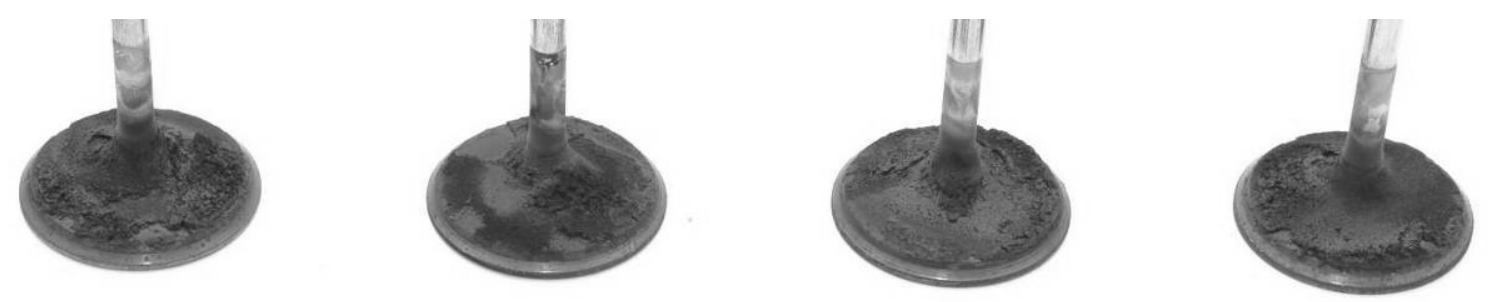

Figura 12. Registro fotográfico das válvulas de admissão após acúmulo de 519 mg.

As figuras 13a e 13b apresentam os registros fotográficos do interior do motor do veículo, antes e após a execução de todo o procedimento de ensaios. As respectivas espessuras médias dos depósitos medidos nas cabeças dos pistões foram de $66 \mu \mathrm{m}$ e $59 \mu \mathrm{m}$, sendo este parâmetro considerado constante durante todo o trabalho. 


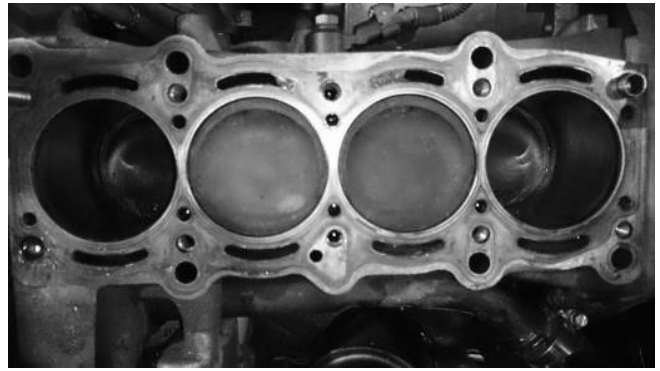

(a)

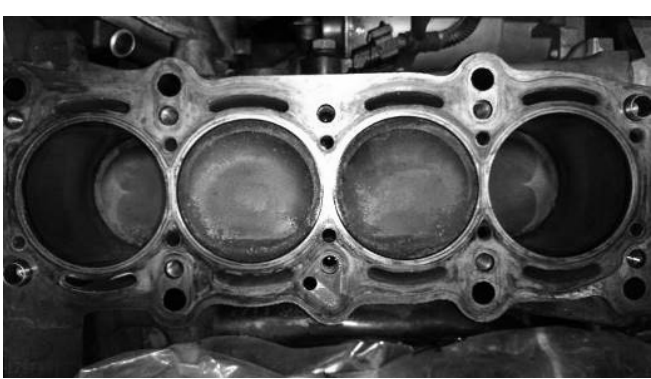

(b)

Figura 13. Registros fotográficos do interior do motor do veículo antes (a) e após (b) a sequência completa de testes.

Nas figuras 14 a 16 e na tabela II estão apresentados os resultados médios de emissões de THC, $\mathrm{CO}$ e NOx obtidos nos ensaios com o motor nas condições de $150 \mathrm{mg}, 340 \mathrm{mg}$ e 519 mg de depósitos acumulados nas válvulas de admissão e seus respectivos valores das incertezas de medição.

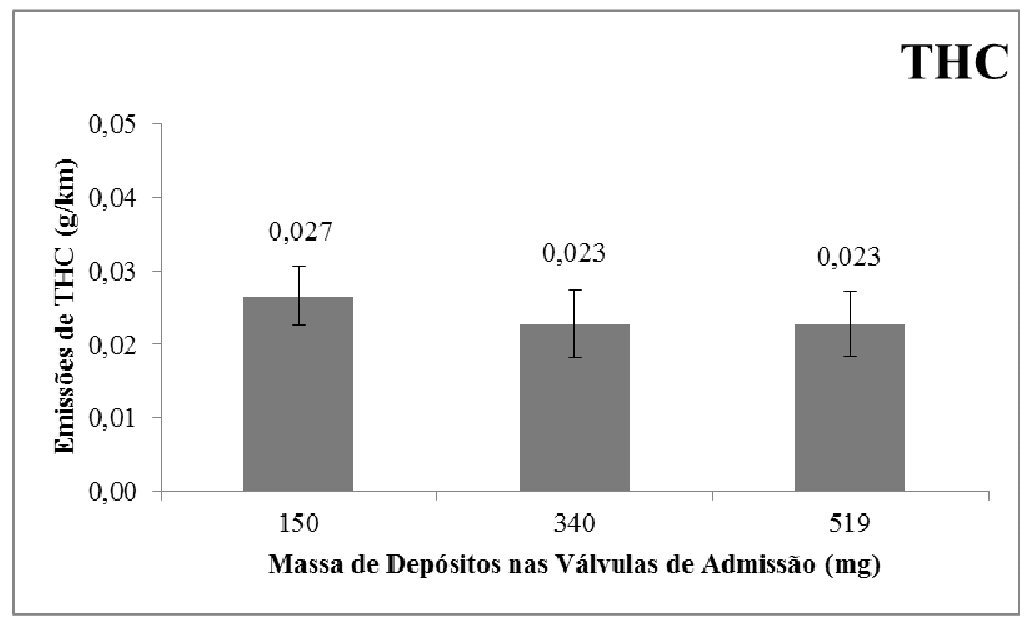

Figura 14. Resultados médios das emissões de THC.

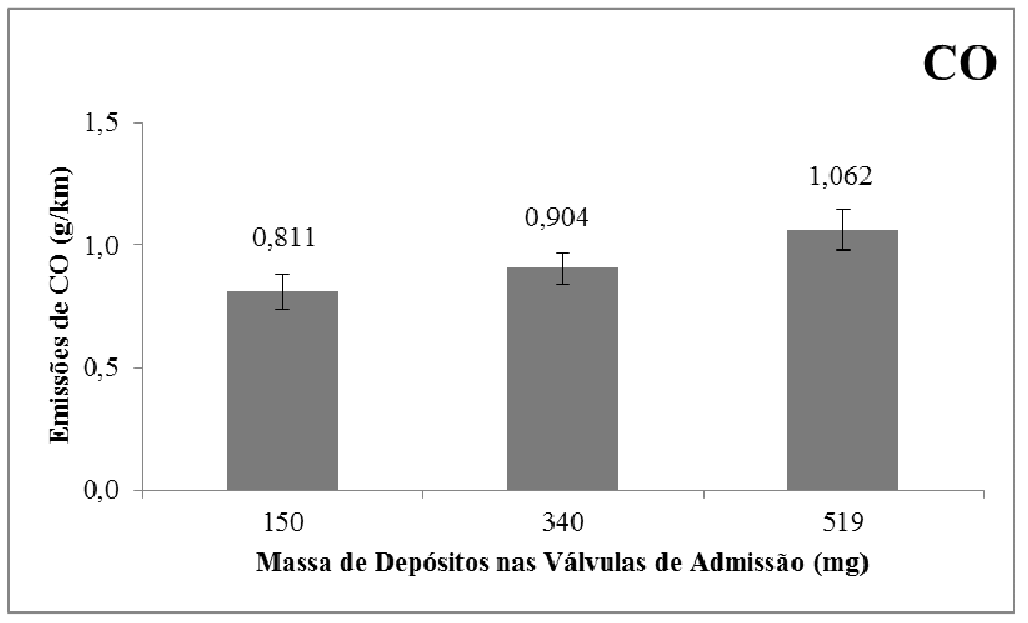

Figura 15. Resultados médios das emissões de CO. 


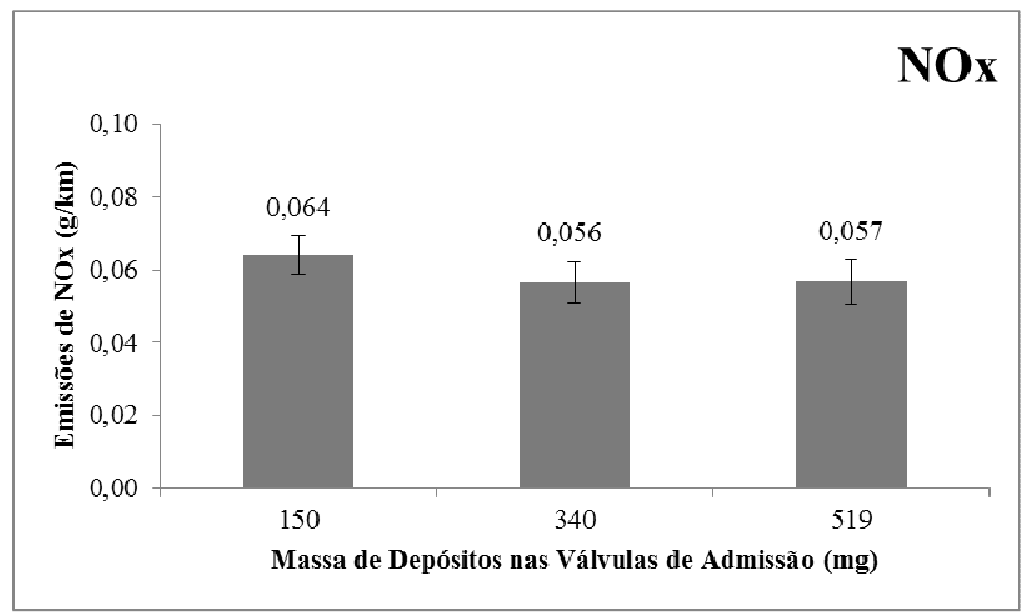

Figura 16. Resultados médios das emissões de NOx.

Tabela II. Resultados médios dos ensaios de emissões de poluentes.

\begin{tabular}{|c|c|c|c|}
\hline $\begin{array}{c}\text { Depósitos } \\
(\mathbf{m g})\end{array}$ & $\begin{array}{c}\text { THC } \\
(\mathbf{g} / \mathbf{k m})\end{array}$ & $\begin{array}{c}\mathbf{C O} \\
(\mathbf{g} / \mathbf{k m})\end{array}$ & $\begin{array}{c}\mathbf{N O x} \\
(\mathbf{g} / \mathbf{k m})\end{array}$ \\
\hline $\mathbf{1 5 0}$ & $0,027 \pm 0,004$ & $0,811 \pm 0,069$ & $0,064 \pm 0,005$ \\
\hline $\mathbf{3 4 0}$ & $0,023 \pm 0,005$ & $0,904 \pm 0,065$ & $0,056 \pm 0,006$ \\
\hline $\mathbf{5 1 9}$ & $0,023 \pm 0,004$ & $1,062 \pm 0,083$ & $0,057 \pm 0,006$ \\
\hline
\end{tabular}

A comparação entre as médias dos resultados dos ensaios de emissões de poluentes realizados com o motor nas condições de 150 e $340 \mathrm{mg}$ de depósitos nas válvulas de admissão é apresentada na tabela III. Foi adotado o teste estatístico $t$ de Student para esta comparação.

Tabela III. Comparação das médias das emissões nas condições de 150 e 340 mg de depósitos nas válvulas de admissão.

\begin{tabular}{|c|c|c|c|c|c|}
\hline \multirow{2}{*}{ Poluentes } & \multicolumn{2}{|c|}{$\begin{array}{c}\text { Médias } \\
(\mathbf{g} / \mathbf{k m})\end{array}$} & \multicolumn{2}{c|}{$\begin{array}{c}\text { Desvios Padrão } \\
(\mathbf{g} / \mathbf{k m})\end{array}$} & \multirow{2}{*}{$\begin{array}{c}\text { t de Student } \\
(\text { valor- } \boldsymbol{~})\end{array}$} \\
\cline { 2 - 5 } & $\mathbf{1 5 0}$ & $\mathbf{3 4 0}$ & $\mathbf{1 5 0}$ & $\mathbf{3 4 0}$ & 0,15 \\
\hline THC & 0,027 & 0,023 & 0,002 & 0,005 & 0,12 \\
\hline CO & 0,811 & 0,904 & 0,092 & 0,074 & 0,06 \\
\hline NOx & 0,064 & 0,056 & 0,004 & 0,007 & 0,06 \\
\hline
\end{tabular}

Como pode ser observado, os resultados do valor-p para os três poluentes obtidos foram maiores do que 0,05 , indicando que não se pode rejeitar a hipótese nula de igualdade estatística entre as médias, o que indica que não há diferença estatisticamente significativa entre elas a um nível de confiança de $95 \%$.

Adicionalmente à comparação entre as médias dos ensaios com $150 \mathrm{mg}$ e $340 \mathrm{mg}$ de depósitos nas válvulas de admissão, foi aplicada a técnica de Análise de Variâncias (ANOVA), considerando-se também as medições de emissões realizadas com o motor na condição de $519 \mathrm{mg}$ de depósitos nas válvulas para verificar a influência do nível de depósitos nas emissões. Na tabela IV são apresentados os resultados. 
Tabela IV. Resultados das Análises de Variância (ANOVA).

\begin{tabular}{|c|c|c|c|c|c|c|c|c|c|}
\hline \multirow{2}{*}{ Grupo } & \multicolumn{3}{|c|}{$\begin{array}{c}\text { THC } \\
(\mathrm{g} / \mathrm{km})\end{array}$} & \multicolumn{3}{|c|}{$\begin{array}{c}\mathrm{CO} \\
(\mathrm{g} / \mathrm{km})\end{array}$} & \multicolumn{3}{|c|}{$\begin{array}{c}\text { NOx } \\
(\mathrm{g} / \mathrm{km})\end{array}$} \\
\hline & Soma & Média & Variância & Soma & Média & Variância & Soma & Média & Variância \\
\hline 150 & 0,133 & 0,027 & 0,0000023 & 4,056 & 0,811 & 0,0083902 & 0,320 & 0,064 & 0,0000185 \\
\hline 340 & 0,114 & 0,023 & 0,0000227 & 4,522 & 0,904 & 0,0055313 & 0,282 & 0,056 & 0,0000433 \\
\hline 519 & 0,114 & 0,023 & 0,0000157 & 5,309 & 1,062 & 0,0189577 & 0,283 & 0,057 & $6,73 \mathrm{E}-05$ \\
\hline valor-P & \multicolumn{3}{|c|}{0,2114} & \multicolumn{3}{|c|}{0,0084} & \multicolumn{3}{|c|}{0,1558} \\
\hline
\end{tabular}

Verifica-se a partir dos resultados obtidos para o valor- $p$ das três análises, que nos casos das emissões de THC e NOx, não há diferenças estatisticamente significativas entre as médias, a um nível de confiança de $95 \%$ (valor- $p>0,05$ ), não caracterizando uma influência dos níveis de depósitos nestas emissões.

No caso das emissões de CO, o valor-p foi inferior a 0,05 indicando a influência dos depósitos nas emissões. Neste caso, foi gerada uma linha de tendência, a partir de regressão linear conforme apresentado na figura 17.

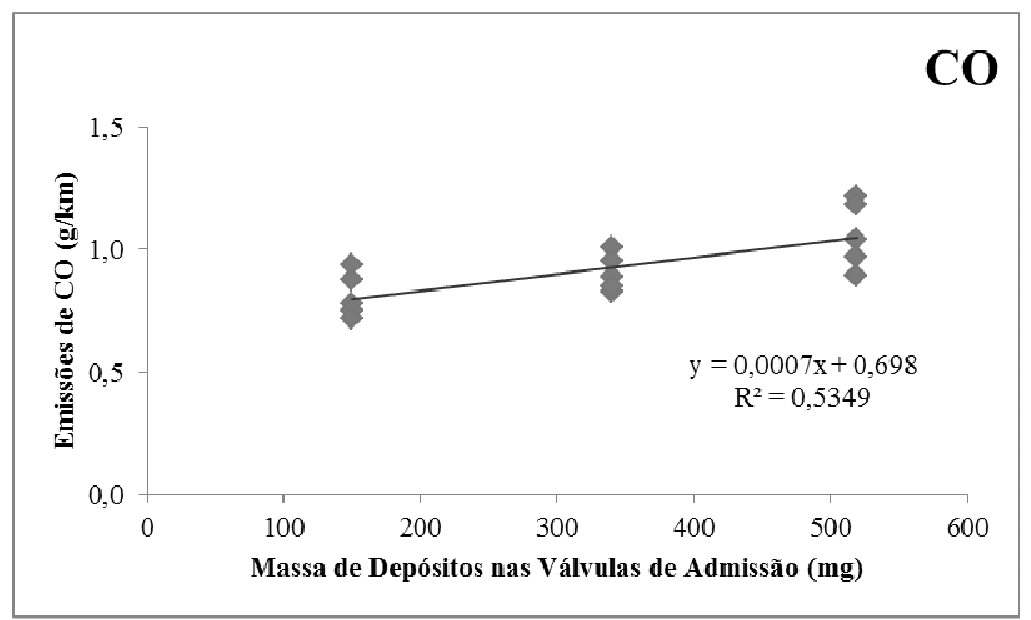

Figura 17. Regressão linear para as emissões de CO.

Adicionalmente à aplicação da técnica ANOVA, para o caso das emissões de CO, foram feitas as comparações entre as médias das medições através do teste $t$ de Student, para o nível de depósitos nas válvulas de admissão de 519 mg em relação aos de $150 \mathrm{mg}$ e $340 \mathrm{mg}$, como apresentado na Tabela V.

Tabela V. Comparações das médias das emissões de CO na condição de 519 mg de depósitos nas válvulas de admissão em relação a 150 e $340 \mathrm{mg}$.

\begin{tabular}{|c|c|c|c|c|c|}
\hline \multirow{2}{*}{ Comparações } & \multicolumn{2}{|c|}{$\begin{array}{c}\text { Médias } \\
(\mathbf{g} / \mathbf{k m})\end{array}$} & \multicolumn{2}{c|}{$\begin{array}{c}\text { Desvios Padrão } \\
(\mathbf{g} / \mathbf{k m})\end{array}$} & $\begin{array}{c}\text { t de Student } \\
(\text { valor-p })\end{array}$ \\
\hline \multirow{2}{*}{$\begin{array}{c}\mathbf{1 5 0} \times \mathbf{5 1 9} \\
(\mathbf{m g})\end{array}$} & $\mathbf{1 5 0}$ & $\mathbf{5 1 9}$ & $\mathbf{1 5 0}$ & $\mathbf{5 1 9}$ & \multirow{2}{*}{$\mathbf{0 , 0 1}$} \\
\cline { 2 - 5 } & 0,811 & 1,062 & 0,092 & 0,138 & \\
\hline \multirow{3}{*}{$\begin{array}{c}\mathbf{3 4 0} \mathbf{x} 519 \\
(\mathbf{m g})\end{array}$} & $\mathbf{3 4 0}$ & $\mathbf{5 1 9}$ & $\mathbf{3 4 0}$ & $\mathbf{5 1 9}$ & \multirow{2}{*}{0,05} \\
\cline { 2 - 5 } & 0,904 & 1,062 & 0,074 & 0,138 & \\
\hline
\end{tabular}


Os resultados das comparações indicam que na comparação entre as condições de $519 \mathrm{mg}$ e $150 \mathrm{mg}$, a hipótese de igualdade entre as médias pode ser rejeitada a um nível de confiança de $95 \%$ (valor-p < 0,05) e assim, houve um aumento de $31 \%$ nas emissões de CO.

Entretanto, na comparação entre as condições de 519 mg e $340 \mathrm{mg}$ não se pode rejeitar a hipótese nula de igualdade estatística entre as médias a um nível de confiança de $95 \%$, o que significa que não há diferença estatisticamente significativa.

A tabela VI resume os resultados das comparações entre as médias das emissões de THC, CO e NOx obtidas nas combinações das três condições de depósitos nas válvulas de admissão. Quando não foram encontradas diferenças estatisticamente significativas, a comparação está indicada com o termo "sem diferença".

Tabela VI. Comparações entre as médias das emissões de poluentes nas combinações das três condições de depósitos em válvulas de admissão ensaiadas.

\begin{tabular}{|c|c|c|c|}
\hline $\begin{array}{c}\text { Comparações } \\
(\mathbf{m g})\end{array}$ & THC & CO & NOx \\
\hline $\mathbf{3 4 0} \times \mathbf{1 5 0}$ & sem diferença & sem diferença & sem diferença \\
\hline $\mathbf{5 1 9} \times \mathbf{3 4 0}$ & sem diferença & sem diferença & sem diferença \\
\hline $\mathbf{5 1 9} \times \mathbf{1 5 0}$ & sem diferença & aumento de $31 \%$ & sem diferença \\
\hline
\end{tabular}

\section{CONCLUSÃO}

O objetivo deste estudo foi gerar informações experimentais a respeito do impacto de diferentes níveis de depósitos em válvulas de admissão de motores nas emissões legisladas de poluentes.

Em função da especificação da ANP para o nível de depósitos a ser gerado por uma gasolina de referência (300 mg) e para o valor de aprovação de gasolinas aditivadas (100 mg), buscouse comparar especificamente o impacto destes dois níveis de depósitos. Adicionalmente, um terceiro nível (500 mg) foi gerado para a verificação de possíveis tendências a partir de técnicas de regressão linear.

$\mathrm{Na}$ literatura pesquisada foram encontrados estudos que indicam o aumento das emissões de THC e CO com o respectivo aumento dos níveis de depósitos nas válvulas de admissão e relacionam as emissões de NOx aos depósitos em câmara de combustão.

Neste trabalho, foi implementado com sucesso um procedimento experimental de testes no qual foram gerados, em banco de provas, os acúmulos de depósitos nas válvulas de admissão, nos níveis desejados (150, 340 e $519 \mathrm{mg}$ ) e posteriormente foram realizados os ensaios de emissões de THC, CO e NOx. Foram aplicadas técnicas estatísticas para comparação das médias duas a duas (teste $t$ de Student) e também considerando as três condições (ANOVA).

Foram utilizados um motor do mesmo modelo especificado na norma NBR 16038, para o qual os limites de depósitos estão sendo estabelecidos, e um veículo equipado com este 
modelo de motor. Não foi avaliada a influência dos depósitos em câmara de combustão, uma vez que durante os testes somente o cabeçote era intercambiado entre o motor do veículo de testes e o instalado no banco de provas.

A partir da amostra ensaiada, não foram observadas diferenças estatisticamente significativas nas emissões de THC e NOx, com o aumento dos níveis dos depósitos nas válvulas de admissão. Em relação às emissões de CO, observou-se que nas comparações "150 x 340 mg" e "340 x 519 mg", não houve diferenças estatisticamente significativas. Somente a variação de $150 \mathrm{mg}$ para $519 \mathrm{mg}$ ocasionou um aumento de cerca de $31 \%$ nas emissões deste poluente.

\section{REFERÊNCIAS}

[1] AGÊNCIA NACIONAL DE PETRÓLEO, GÁS NATURAL E BIOCOMBUSTÍVEIS, Resolução ANP nº 40, 25 de outubro de 2013.

[2] AGÊNCIA NACIONAL DE PETRÓLEO, GÁS NATURAL E BIOCOMBUSTÍVEIS, Resolução ANP n ${ }^{\circ}$ 1, 7 de janeiro de 2014.

[3] ASSOCIAÇÃO BRASILEIRA DE NORMAS TÉCNICAS, "Combustíveis — Medição de Depósitos em Válvulas de Admissão em Motor com Ignição por Centelha", NBR 16038, setembro de 2012.

[4] ASSOCIAÇÃO BRASILEIRA DE NORMAS TÉCNICAS, "Determinação de Hidrocarbonetos, Monóxido de Carbono, Óxidos de Nitrogênio e Dióxido de Carbono no Gás de Escapamento" NBR 6601, setembro de 2012.

[5] HOUSER, K. R., CROSBY, T. A., "The Impact of Intake Valve deposits on Exhaust Emissions", SAE Technical Paper 922259.

[6] ZAHALKA, T. L., KULINOWSKI, A. M., MALFER, D. J., "A Fleet Evaluation of IVD and CCD: Emissions effects and Correlation to the BMW 318i and Ford 2.3L IVD Tests", SAE Technical Paper 952447.

[7] BitTing, W. H., FIRMSTONE, G. P., KELLER, C. T., "Effects of Combustion Chamber Deposits on Tailpipe Emissions", SAE Technical Paper 940345.

[8] BARNES, J. R., STEPHENSON, T., "Influence of Combustion Chamber Deposits on Vehicle Performance and Tailpipe Emissions", SAE Technical Paper 962027.

[9] ARTERS, D. C., BARDASZ, E. A., SCHIFERL, E. A. et al., "A Comparison of Gasoline Direct Injection and Port Fuel Injection Vehicles; Part I - Fuel System Deposits and Vehicle Performance", SAE Technical Paper 1999-01-1498.

[10] SANDQUIST, H, DENBRATT, I., OWRANG, F. et al., "Influence of Fuel Parameters on Deposit Formation and Emissions in a Direct Injection Stratified Charge SI Engine", SAE Technical Paper 2001-01-2028.

[11] RAMADHAS, A. S., SINGH, V. P., SUBRAMANIAN, M. et al., "Impact of Fuel Additives on Intake Valve Deposits, Combustion Chamber Deposits and Emissions", SAE Technical Paper 2011-01-1980.

[12] YUE, X., WU, Y., HUANG, X. et al., "Impact of Gasoline Deposits on Light Duty Vehicle Emissions: In-use Case Study in Beijing, China, Front. Environ. Sci. Eng. 2012, 6(5): 717-724. DOI 10.1007/s11783-012-0438-3. 